\title{
Thoughts on the Concept of Stress
}

\author{
Walter Noll
}

January 13, 2009

Note: The mathematical infrastructure, notation, and terminology employed here can be found in [FDS]. For example, the set of real numbers is denoted by $\mathbf{R}$ and the set of strictly (non-zero) real numbers by $\mathbb{P}^{\times}$.

In Section 5 below we use the notation for volume integrals as explained in Chapter 4 of Vol.II of [FDS]. In particular, let a mapping $\mathbf{h}: \mathcal{D} \longrightarrow \mathcal{W}$ be given whose domain $\mathcal{D}$ is a region in a Euclidean space, a vector space, or the real line, and whose codomain $\mathcal{W}$ is a linear space. Then, the volume integral of $\mathbf{h}$ over a suitable subregion $\mathcal{P}$ of $\mathcal{D}$, if meaningful, is denoted by $\int_{\mathcal{P}} \mathbf{h}$ or $\int_{\mathcal{P}} \mathbf{h}(x) \mathrm{d} x$. In the latter case, the $\mathrm{d} x$ should be read as "the dummy is $x$ " and it should be used only if the mapping $\mathbf{h}$ is not described by a single symbol but only by symbols for its values. In this case, the letter $x$ can be replaced by any other letter without change of meaning, provided only that this letter is not used for other purposes in the same context. For surface integrals, we use $\mathrm{d}_{\mathrm{ar}} z$ instead of just $\mathrm{d} z$ to say that we deal with surface-area integration and that the dummy is $z$.

\section{Materially Ordered Sets}

Stress is not a primitive concept; it is based on the concept of force, which can be described by a basic mathematical structure and then is connected to real situations by constitutive laws, laws which may be internal or external. To deal with force one first has to describe what is meant by a physical system.

The concept of a materially ordered set was first introduced by me in the context of an axiomatic foundation of physical systems (see [N 1]). Here $\Omega$, as defined below and in [NS], is considered to consist of the whole system and all of its parts. Given $a, b \in \Omega, a \prec b$ is read " $a$ is a part of $b$ ". The maximum ma is the "material all", i.e., the whole system, and the minimum $\mathrm{mn}$ is the "material nothing". The $\inf \{a, b\}$ is the "common part" of $a$ and $b$, and $a^{\mathrm{rem}}$ is the part of the whole system ma that remains after $a$ has been removed. With this in mind, the two conditions (MO3) and (MO4) below are very natural.

Definition 1. An ordered set $\Omega$ with order $\prec$ is said to be materially ordered if the following axioms are satisfied:

(MO1) $\Omega$ has a maximum ma and a minimum mn.

(MO2) Every doubleton has an infimum.

(MO3) For every $a \in \Omega$ there is exactly one member of $\Omega$, denoted by $a^{\mathrm{rem}}$. such that $\inf \left\{a, a^{\mathrm{rem}}\right\}=\mathrm{mn}$ and $\sup \left\{a, a^{\text {rem }}\right\}=$ ma.

(MO4) $\left(\inf \left\{a, b^{\mathrm{rem}}\right\}=\mathrm{mn}\right) \Longrightarrow a \prec b \quad$ for all $\quad a, b \in M$. 
The mapping rem $:=\left(a \mapsto a^{\mathrm{rem}}\right): \Omega \leftrightarrow \Omega$ is called the remainder mapping in $\Omega$.

Theorem 1: Let $\Omega$ be a materially ordered set and $p \in \Omega$ be given. Then $\Omega_{p}:=\{q \in \Omega \mid q \prec p\}$ is a materially ordered set and the remainder mapping in $\Omega_{p}$ is given by

$$
\operatorname{rem}_{p}:=\left(a \mapsto a^{\mathrm{rem}} \wedge p\right) .
$$

The proof is fairly trivial.

Theorem 2: Let $\Omega$ be a materially ordered set. Then $\Omega$ has the structure of a Boolean algebra ${ }^{1}$ with

$$
\operatorname{meet}(a, b):=\inf \{a, b\} \text { and } \operatorname{cpt}(a):=a^{\text {rem }} \quad \text { for all } a, b \in \Omega, \quad \text { and } \quad \text { tp }:=\text { ma }
$$

The proof is very non-trivial. The best version of it is given in [NS].

\section{Interactions}

As was noted in the previous section, materially ordered sets are models for physical systems.

Let $\Omega$ be a materially ordered set, and let $\mathcal{W}$ be a linear space. We say that the parts $p$ and $q$ are separate if $p \wedge q=\mathrm{mn}$. We use the notation

$$
\left(\Omega^{2}\right)_{\operatorname{sep}}:=\left\{(p, q) \in \Omega^{2} \mid p \wedge q=\mathrm{mn}\right\} .
$$

Definition 2. A function $H: \Omega \longrightarrow \mathcal{W}$ is said to be additive if

$$
H(p \vee q)=H(p)+H(q) \text { for all }(p, q) \in\left(\Omega^{2}\right)_{\operatorname{sep}} .
$$

A function $I:\left(\Omega^{2}\right)_{\text {sep }} \longrightarrow \mathcal{W}$ is said to be an interaction in $\Omega$ if, for all $p \in \Omega$, both $I\left(\cdot, p^{\mathrm{rem}}\right): \Omega_{p} \longrightarrow \mathcal{W}$ and $I\left(p^{\mathrm{rem}}, \cdot\right): \Omega_{p} \longrightarrow \mathcal{W}$ are additive.

The resultant $R_{I}: \Omega \rightarrow \mathcal{W}$ of a given interaction $I$ in $\Omega$ is defined by

$$
\mathrm{R}_{I}(p):=I\left(p, p^{\mathrm{rem}}\right) \text { for all } p \in \Omega \text {. }
$$

We say that a given interaction is skew if

$$
I(q, p)=-I(p, q) \text { for all }(p, q) \in\left(\Omega^{2}\right)_{\operatorname{sep}}
$$

Remark 1: The concept of an interaction is an abstraction. Its values may have the interpretation of forces, torques, or heat transfers. In most of the past literature these cases were treated separately even though much of the underlying mathematics is the same for all. Thus, this abstraction, like most others, is a labor saving device. I

\footnotetext{
${ }^{1}$ We use the notations that occur in the definition of a Boolean Algebra in Section 2 of $[\mathrm{NS}]$, which include meet $=\wedge$, join $=\vee, \operatorname{ctp}$ (counterpart), and tp (top).
} 
Theorem 3: An interaction is skew if and only if its resultant is additive.

The proof, based on the Boolean algebra structure of $\Omega$ described by Theorem 2, is fairly simple and it is given, for example, in Section 4 of [NS].

Remark 2: Theorem 3 states that the law of action and reaction, which is referred to as Newton's Third Law when the interactions are interpreted as forces, is true if and only if the resultant is additive. The fact that the resultant caused by the interaction is additive is a consequence of basic balance laws. Thus, if one assumes appropriate balance laws, one can prove Newton's Third Law instead of assuming it. Such a balance law can be formulated as follows: "The resultant of a given interaction is balanced by the additive action of the exterior world on the system and its parts."

\section{Fit regions}

We consider the following problem: What type of subsets of Euclidean spaces are "fit" to be regions occupied by a continuous body in a frame of reference?

After some thought, I came to the conclusion, long ago, that the class of all such fit regions should satisfy the following requirements:

A fit region should

(a) be an open set (possible domain of $\mathrm{C}^{1}$ - mappings),

(b) have a boundary that is also the boundary of its exterior (no "hidden" boundary points),

(c) have an exterior normal at all but "exceptional" boundary points, and the Integral-Gradient Theorem (Divergence Theorem) should be applicable.

(d)The class of all fit regions should be invariant under whole-space $\mathrm{C}^{2}$-diffeomorphisms.

(e)The fit regions included in a given one should be materially ordered by inclusion.

Ideally, the class of all fit regions should include all that can be possibly imagined by an engineer but exclude those that only an ingenious mathematician can think of. It is about 30 years ago that I reached the conclusion that there is no such class. I now believe that the best one can do is use the class Fr of fit regions proposed in [NV 1], or minor variations thereof. This class consist of all sets that are regularly open, are bounded, have a negligible boundary, and have a finite perimeter.

Let a Euclidean space $\mathcal{E}$ with translation space $\mathcal{V}$ be given. The set of all fit regions in $\mathcal{E}$ is denoted by $\operatorname{Fr}(\mathcal{E})$. For every $\mathcal{P} \in \operatorname{Fr}(\mathcal{E})$, $\operatorname{Rby}(\mathcal{P})$ denotes the reduced boundary of $\mathcal{P}$, i.e., the set of all boundary points of $\mathcal{P}$ at which a unit normal can be defined, leaving out all boundary points on edges, cusps, etc. We use the notation

$$
\mathbf{n}_{\mathcal{P}}: \operatorname{Rby}(\mathcal{P}) \longrightarrow \operatorname{Usph}(\mathcal{V}):=\{\mathbf{v} \in \mathcal{V}|| \mathbf{v} \mid=1\}
$$

for the function that assingns to each $z \in \operatorname{Rby}(\mathcal{P})$ the unit normal $\mathbf{n}_{\mathcal{P}}(z)$ directed away from $\mathcal{P}$.

The proof of the folloing theorem, given in [NV 1], is extremely non-trivial. 
Theorem 4: Let $\mathcal{B} \in \operatorname{Fr}(\mathcal{E})$ be given and put

$$
\Omega:=\operatorname{Fr} \mathcal{B}:=\{\mathcal{P} \in \operatorname{Fr}(\mathcal{E}) \mid \mathcal{P} \subset \mathcal{B}) .
$$

Then $\Omega$ is materially ordered by inclusion with $\mathrm{mn}:=\emptyset$, ma $:=\mathcal{B}$.

In this case, given $\mathcal{P} \in \operatorname{Fr} \mathcal{B}$ we use the term remainder relative to $\mathcal{B}$ and denote it by $\mathcal{P}^{\mathcal{B}}$ rather than $\mathcal{P}^{\text {rem }}$. For all $\mathcal{P}, \mathcal{Q} \in \Omega$ we have ${ }^{2}$

$$
\begin{gathered}
\mathcal{P}^{\mathcal{B}}=\operatorname{Int}(\mathcal{B} \backslash \mathcal{P}), \\
\mathcal{P} \wedge \mathcal{Q}=\mathcal{P} \cap \mathcal{Q}, \\
\mathcal{P} \vee \mathcal{Q}=\operatorname{Int} \operatorname{Clo}(\mathcal{P} \cup \mathcal{Q})
\end{gathered}
$$

\section{Surface interactions}

Again, we assume that a Euclidean space $\mathcal{E}$ with translation space $\mathcal{V}$ and $\mathcal{B} \in$ Fr $\mathcal{E}$ are given, and we consider the materially ordered set $\Omega:=\operatorname{Fr} \mathcal{B}$ as described by Theorem 4 .

Definition 9: We say that a given interaction $I$ in $\Omega$ with values in a given linear space $\mathcal{W}$ is a surface interaction if

$$
\operatorname{area}(\operatorname{Rct}(\mathcal{P}, \mathcal{Q}))=0 \Longrightarrow I(\mathcal{P}, \mathcal{Q})=\mathbf{0} \quad \text { for all } \quad(\mathcal{P}, \mathcal{Q}) \in\left(\Omega^{2}\right)_{\text {sep }}
$$

Where $\operatorname{Rct}(\mathcal{P}, \mathcal{Q}):=\operatorname{Rby}(\mathcal{P}) \cap \operatorname{Rby}(\mathcal{Q})$ is the reduced contact of $\mathcal{P}$ and $\mathcal{Q}$.

Remark 3: Not every contact interaction, as defined in Section 4 of [NV 2], is a surface interaction. The edge interactions discussed in [NV 2] are contact interactions but not surface interactions. They may be relevant when dealing with surface tension.

Theorem 5: When suitable regularity assumptions are made, a given surface interaction $I$ is determined by a contactor $\mathbf{C}: \mathcal{B} \longrightarrow \operatorname{Lin}(\mathcal{V}, \mathcal{W})$ with the property that

$$
I(\mathcal{P}, \mathcal{Q})=\int_{\operatorname{Rct}(\mathcal{P}, \mathcal{Q})} \mathbf{C}(z) \mathbf{n}_{\mathcal{P}}(z) \mathrm{d}_{\mathrm{ar}}(z) \quad \text { for all } \quad(\mathcal{P}, \mathcal{Q}) \in\left(\Omega^{2}\right)_{\mathrm{sep}}
$$

where $\mathbf{n}_{\mathcal{P}}$ is the exterior unit normal to $\mathcal{P}$ as described by (3.1) in the previous section.

To this day, it is not quite clear what regularity assumption must be made to validate the conclusion of Theorem 5. In any case, the proof is extremely non-trivial.

Remark 4: There is a very large literature on surface interactions, going back to Cauchy and Fourier in the early 19th century. In [N 2] I describe what I believe to be the present state of this analysis.

Remark 5: In the case when the interaction is a force, the contactor $\mathbf{T}: \mathcal{B} \longrightarrow \operatorname{Lin}(\mathcal{V})$ is the contact stress. It the case when the interaction is heat transfer, the contactor $\mathbf{q}: \mathcal{B} \longrightarrow \operatorname{Lin}(\mathcal{V}, \mathbf{R}) \cong \mathcal{V}$ is the heat flux .

\footnotetext{
${ }^{2}$ We use the notations Int and Clo for the processes of forming the interior and the closure of a subset of a topological space.
} 


\section{Volume Interactions}

Again, we assume that a Euclidean space $\mathcal{E}$ with translation space $\mathcal{V}$ and $\mathcal{B} \in \operatorname{Fr} \mathcal{E}$ are given, and we consider the materially ordered set $\Omega:=\operatorname{Fr} \mathcal{B}$ as described by Theorem 4 . In addition, let a linear space $\mathcal{W}$ be given.

Definition 1. We say that an interaction $J:\left(\Omega^{2}\right)_{s e p} \longrightarrow \mathcal{W}$ is a volume interaction if, for some norm $\nu$ on $\mathcal{W}$, there is $c \in \mathbb{P}^{\times}$such that

$$
\nu(J(p, \mathcal{Q})) \leq c \operatorname{vol}(p) \operatorname{vol}(\mathcal{Q}) \quad \text { for all } \quad(p, \mathcal{Q}) \in\left(\Omega^{2}\right)_{s e p} .
$$

If $\mathcal{W}$ is finite dimensional, then, by the norm-equivalence theorem, "for some norm" in Definition 1 can be replaced by "for all norms".

We assume now that such a volume interaction is given and that it is skew.

Theorem 6. If certain regularity conditions are satisfied there is exactly one integrable function $\mathrm{g}: \mathcal{B} \times \mathcal{B} \longrightarrow \mathcal{W}$, called the interaction density such that

$$
\mathbf{g}(x, y)=-\mathbf{g}(y, x) \quad \text { for all } \quad x, y \in \mathcal{B}
$$

and

$$
J(\mathcal{P}, \mathcal{Q})=\int_{\mathcal{P} \times \mathcal{Q}} \text { g for all } \quad(\mathcal{P}, \mathcal{Q}) \in\left(\Omega^{2}\right)_{\text {sep }}
$$

By the Iterated Integral Theorem we then have

$$
J(\mathcal{P}, \mathcal{Q})=\int_{\mathcal{Q}}\left(\int_{\mathcal{P}} \mathbf{g}(x, y) \mathrm{d} x\right) \mathrm{d} y=\int_{\mathcal{P}}\left(\int_{\mathcal{Q}} \mathbf{g}(x, y) \mathrm{d} y\right) \mathrm{d} x \quad \text { for all } \quad(\mathcal{P}, \mathcal{Q}) \in\left(\Omega^{2}\right)_{\text {sep }} .
$$

We now assume that a function $\mathbf{g}: \mathcal{B} \times \mathcal{B} \longrightarrow \mathcal{W}$ has been determined according to Theorem 6 .

Definition 2. A function $\mathbf{R}: \mathcal{B} \longrightarrow \operatorname{Lin}(\mathcal{V}, \mathcal{W})$ is called a reacher for $\mathbf{g}$ if, for all $\mathcal{P} \in \Omega$, we have

$$
J\left(\mathcal{P}, \mathcal{P}^{\mathcal{B}}\right)=\int_{\mathcal{P} \times \mathcal{P} \mathcal{B}} \mathbf{g}=\int_{\operatorname{Rby}(\mathcal{P})} \mathbf{R}(z) \mathbf{n}_{\mathcal{P}}(z) \mathrm{d}_{\text {ar }}(z) \quad \text { for all } \mathcal{P} \in \Omega
$$

Without change of notation, we now extend the domain of $\mathbf{g}$ to $\mathcal{E} \times \mathcal{E}$ by putting

$$
\mathbf{g}(x, y)=\mathbf{0} \quad \text { for all } \quad(x, y) \notin \mathcal{B} \times \mathcal{B} .
$$

Theorem 7. If $\mathbf{g}$ satisfies suitable regularity conditions, then $\mathbf{R}: \mathcal{B} \longrightarrow \operatorname{Lin}(\mathcal{V}, \mathcal{W})$, defined by ${ }^{3}$

$$
\mathbf{R}(z):=-\frac{1}{2} \int_{\mathcal{V}}\left(\int_{[0,1]}(\mathbf{g} \circ(z+\iota \mathbf{v}, z-(1-\iota) \mathbf{v})) \otimes \mathbf{v}\right) \mathrm{d} \mathbf{v} \quad \text { for all } z \in \mathcal{B}
$$

is a reacher for $\mathbf{g}$.

\footnotetext{
${ }^{3} \iota$ denotes the identity mapping of $\mathbf{R}$.
} 
Assuming that the extended function $\mathrm{g}: \mathcal{E} \times \mathcal{E} \longrightarrow \mathcal{W}$ is of class $\mathrm{C}^{1}$, we give the steps toward a proof. The justification for each of these steps is left to the reader. The proof is a modified and updated version of a proof given in Section 9 of [N 3].

Let $y \in \mathcal{E}$ and $\mathbf{v} \in \mathcal{V}$ be given.

Step 1: We have

$$
\int_{\mathcal{E}} \mathbf{g}(\cdot, y)=\frac{1}{2} \int_{\mathcal{V}}(\mathbf{g}(y+\mathbf{v}, y)-\mathbf{g}(\mathbf{v}, y-\mathbf{v})) \mathrm{d} \mathbf{v}
$$

Step 2: We have, for all $\alpha \in \mathbf{R}$,

$$
\nabla_{y}(z \mapsto \mathbf{g}(z-(1-\alpha) \mathbf{v}, z+\alpha \mathbf{v}))=\nabla_{(1)} \mathbf{g}\left((y-(1-\alpha) \mathbf{v}, y+\alpha \mathbf{v})+\nabla_{(2)} \mathbf{g}(y-(1-\alpha) \mathbf{v}, y+\alpha \mathbf{v}) \in \operatorname{Lin}(\mathcal{V}, \mathcal{W})\right.
$$

Step 3: We have ${ }^{4}$

$$
(\mathbf{g} \circ(y-(1-\iota) \mathbf{v}, y+\iota \mathbf{v}))^{\bullet}=\left(\nabla_{y}(z \mapsto \mathbf{g} \circ(z-(1-\iota) \mathbf{v}, z+\iota \mathbf{v}))\right) \mathbf{v} .
$$

Step 4: Integration of (5.10) from 0 to 1 and using the Fundamental Theorem of Calculus gives

$$
\mathbf{g}(y+\mathbf{v}, y)-\mathbf{g}(\mathbf{v}, y-\mathbf{v})=\nabla_{y}\left(z \mapsto\left(\int_{[0,1]} \mathbf{g} \circ(z-(1-\iota) \mathbf{v}, z+\iota \mathbf{v})\right) \mathbf{v}\right)
$$

Step 5: Using (5.8), (5.11), and Definition 1 of Section 64 of [FDS], Vol.I, we obtain

$$
\int_{\mathcal{E}} \mathbf{g}(\cdot, y)=\operatorname{div}_{y} \mathbf{R} \in \mathcal{W} \quad \text { for all } \quad y \in \mathcal{E}
$$

Step 6: Let $\mathcal{P} \in \Omega$ be given. Using the DivergenceTheorem, it follows from (5.12) that

$$
\int_{\mathcal{P} \times \mathcal{E}} \mathbf{g}=\int_{\mathcal{P}}\left(\int_{\mathcal{E}} \mathbf{g}(\cdot, y)\right) \mathrm{d} y=\int_{\operatorname{Rby}(\mathcal{P})} \mathbf{R}(z) \mathbf{n}^{\mathcal{P}}(z) \mathrm{d}_{\mathrm{ar}}(z) .
$$

Since $\int_{\mathcal{P} \times \mathcal{E}} \mathbf{g}=\int_{\mathcal{P} \times \mathcal{P}} \mathbf{g}+\int_{\mathcal{P} \times \mathcal{P}} \mathbf{g}$ and since $\int_{\mathcal{P} \times \mathcal{P}} \mathbf{g}=\mathbf{0}$ by (5.2), the desired result (5.5) follows.

If the extended function $\mathbf{g}$ is not of class $\mathrm{C}^{1}$ but satisfies suitable conditions, the assertion (5.5) with $\mathbf{R}$ defined by (5.7) can still be proved by using limiting arguments.

\section{Forces and Stresses}

We assume that a Euclidean space $\mathcal{E}$ with translation space $\mathcal{V}$ and $\mathcal{B} \in$ Fr is given, define $\Omega$ by (3.2), and consider the corresponding physical system.

A system of external forces on the system is a mapping $E: \Omega \longrightarrow \mathcal{V}$, so that $E(\mathcal{P})$ denotes the external force acting on a given part $\mathcal{P} \in \Omega$, We assume that the external forces acting on the system are volume forces

\footnotetext{
${ }^{4}$ To honor Newton, we use the superscript ${ }^{\bullet}$ for derivatives.
} 
with a density $\mathbf{b}: \mathcal{B} \longrightarrow \mathcal{V}$, so that

$$
E(\mathcal{P})=\int_{\mathcal{P}} \mathbf{b} \quad \text { for all } \mathcal{P} \in \Omega
$$

Inertial forces are always considered to be external.

An interaction in $\Omega$ with values in $\mathcal{V}$ can now be viewed as a system of internal forces. Let $I$ be is surface interaction which can be described by a contactor as in Theorem 5. In this case, the contactor is a lineon-field $\mathbf{T}: \mathcal{B} \longrightarrow \operatorname{Lin}(\mathcal{V})^{5}$ which should be called the contact stress. It follows from the law of balance of torques that the values of $\mathbf{T}$ are symmetric and hence that $\operatorname{Rng} \mathbf{T} \subset \operatorname{Sym} \mathcal{V}$. If $I$ is the only interaction in $\Omega$ and if the contact stress is differentiable, then the law of balance of forces reduces to the form

$$
\operatorname{div} \mathbf{T}+\mathbf{b}=\mathbf{0} .
$$

This is the basic balance law one can find in every textbook on continuum mechanics for engineers.

In engineering, internal volume forces are usually considered to be negligible. When building a bridge, the gravitational attraction between the various parts of the bridge, in contrast to the external gravitational attraction by the earth, is too small to make any difference. However, when the system is an entire star or planet, internal gravitational attraction is very important. Also, internal electrical or magnetic forces may be important even in much smaller systems. Let us assume, then, that the internal force system is the valuewise sum of a surface interaction $I$ and a skew volume interaction $J$. Assume that the volume interaction has a density $\mathbf{g}: \mathcal{B} \times \mathcal{B} \longrightarrow \mathcal{V}$ as described by Theorem 6 . It follows from the law of balance of torques and also from the principle of frame-indifference (see Section 4 of Part 2 of $[\mathrm{FC}]$ ) that $\mathbf{g}(x, y)$ must be in the direction of $x-y$. Hence there is a function $\gamma: \mathcal{B} \times \mathcal{B} \longrightarrow \mathbf{R}$ such that

$$
\mathbf{g}(x, y)=\gamma(x, y)(x-y) \text { and } \quad \gamma(x, y)=\gamma(y, x) \quad \text { for all } \quad x, y \in \mathcal{B} .
$$

In this case, the reacher given by (5.7) reduces to

$$
\mathbf{R}(z) ;=-\frac{1}{2} \int_{\mathcal{V}}\left(\int_{[01]}(\gamma(z+\iota \mathbf{v}, z-(1-\iota) \mathbf{v}))(\mathbf{v} \otimes \mathbf{v}) \mathrm{d} \mathbf{v} \quad \text { for all } z \in \mathcal{B}\right.
$$

and hence has symmetric values. It may be called the reaching stress for the given volume interaction.

If the volume interaction is gravitational or electrostatic, the function $\gamma$ has the form

$$
\gamma(x, y)=c \frac{\sigma(x) \sigma(y)}{|x-y|^{3}} \quad \text { for all } \quad x, y \in \mathcal{B},
$$

where $\sigma: \mathcal{B} \longrightarrow \mathbf{R}$ is the mass density or electrical charge density, respectively. $c$ is a universal constant in each case. The balance equation (6.2) must now be replaced by

$$
\operatorname{div}(\mathbf{T}+\mathbf{R})+\mathbf{b}=\mathbf{0} .
$$

\section{Final remarks}

One may consider the possibility that contact stresses can be viewed as limits of reaching stresses when the support of $\mathbf{g}$ shrinks to the diagonal $\{\{x, x\} \mid x \in \mathcal{B}\}$ of $\mathcal{B} \times \mathcal{B}$. I believe that this is realistic only in special cases.

\footnotetext{
${ }^{5}$ The members of Lin $(\mathcal{V})$ are called "linear transformations" or lineons for short.
} 
The reaching stress $\mathbf{R}$, with $\gamma$ given by (6.5) is of great importance in applications of continuum physics to astrophysics, where internal gravitational forces are crucial. In this case the internal gravitational force density $\operatorname{div} \mathbf{R}$ is also the gradient of the solution of an appropriate Poisson equation.

Electromagnetic interactions are more complicated to analyze because they involve not only force interactions but also torque interactions at a distance and by contact, and hence also body-couples and couple-stresses. Moreover, when dealing with induction, one must consider not only the positions of the material points but also their relative motion.

I believe that the relation between continuum mechanics and statistical mechanics described in [N 3] is realistic only in very special cases. In general, statistical considerations based on quantum mechanics would be needed. The connection of the present paper with [N 3] is Theorem 7 above and the two Lemmas in Section 9 of [N 3].

\section{References:}

[FDS] Noll, W., Finite-Dimensional Spaces: Algebra, Geometry, and Analysis, Vol.I and Vol.II, published on the website math.cmu.edu/ wnog/noll . ( Vol.I was published by Martinus Nijhoff Publishers in 1987.) Vol.I has 393 pages. The preliminary manuscript of Vol.II has about 110 pages and is growing.

[FC] Noll, W., Five Contributions to Natural Philosophy, 73 pages, (2004), published on the website math.cmu.edu/ wnog/noll .

[N 1] Noll, W., Lectures on the Foundations of Continuum Mechanics and Thermodynamics, Archive for Rational Mechanics and Anaysis 52, 62-92 (1973). (An earlier version was presented in 1965 in a published lecture.)

[N 2] Noll, W., On the Theory of Surface Interactions (2005), 14 pages, published on the website math.cmu.edu/ wnOg/noll .

[N 3] Noll, W., Die Herleitung der Grundgleichungen der Thermomechanik der Kontinua aus der Statistischen Mechanik, Journal of Rational Mechanics and Analysis 4, 627-646 (1955). Recently, Anatole Lilienfeld and Rich Lahouc provided an English translation.

[NS] Noll, W. \& Seguin, B., Monoids, Boolean Algebras, Materially Ordered Sets, International Journal of Pure and Applied Mathematics 37, 187-202 (2007), also published on the website math.cmu.edu/ wnog/noll.

[NV 1] Noll, W. \& Virga, E., Fit Regions and Functions of bounded variation, Archive for Rational Mechanics and Analysis 102, 1-21 (1988).

[NV 2] Noll, W. \& Virga, E., On edge interactions and surface tension, Archive for Rational Mechanics and Analysis 111, 1-31 (1990). 